\title{
DEVELOPMENT AND EVALUATION OF CLOBETASOL-LOADED SOLID LIPID NANOPARTICLES FOR TOPICAL TREATMENT OF PSORIASIS
}

\section{K. RAMESH REDDY ${ }^{1}$, S. V. SATYANARAYANA ${ }^{2}$, V. JAYASANKAR REDDY ${ }^{*}$}

${ }^{1}$ Department of Pharmaceutical Sciences, Jawaharlal Nehru Technological University Anantapur, Ananthapuramu, Krishna Teja Pharmacy College, Chadalawada Nagar, Tirupati, Andhra, Pradesh, India, ${ }^{2}$ Department of Chemical Engineering, Jawaharlal Nehru Technological University, Anantapur, Ananthapuramu, Andhra Pradesh, India, ${ }^{3}$ Department of Pharmacology, Krishna Teja Pharmacy College, Chittoor, Andhra Pradesh, India

Email: k.rameshreddy88@gmail.com

Received: 13 Apr 2019, Revised and Accepted: 04 Jul 2019

\section{ABSTRACT}

Objective: The current research was structured to achieve a maximum topical delivery for the drug clobetasol-17-propionate (CP) and to predict the effects of various independent variables like lipid: drug ratio, surfactant, and homogenization time on particulate characters and performance solid lipid nanoparticles (SLNs).

Methods: CP loaded SLNs were formulated by Emulsification-Homogenization method and optimized using $3^{3}$ full factorial designs (Design-Expert software 11.0). Drug loaded SLNs were evaluated for various parameters like particle size, surface charge, polydispersity index, entrapment efficiency, surface morphology, thermal analysis, in vitro drug release through skin (Franz diffusion cell), drug deposition study and stability.

Results: The optimized formulation (SLNs) attains a minimal Particle size of $133.3 \pm 3.66 \mathrm{~nm}$, Poly dispersibility index of $0.179 \pm 0.081, \%$ entrapment efficiency of $78.1 \pm 1.11$ and Zeta potential of- $36.2 \pm 0.11 \mathrm{mV}$. Skin permeation study of CP loaded SLNs suspension showed prolonged drug release up to $24 \mathrm{~h}$. Maximum drug deposition was obtained after developing the drug into SLNs $(48.22 \mu \mathrm{g} / \mathrm{ml})$ when compared to the pure drug $(19.12 \mu \mathrm{g} / \mathrm{ml})$.

Conclusion: SLNs were promising colloidal particulate carriers by which prolonged drug release and improved skin permeation was achieved for the drug Clobetasol 17- propionate.

Keywords: Clobetasol-17-propionate, Solid lipid nanoparticles, $3^{3}$ full factorial design, Franz diffusion cell

(C) 2019 The Authors. Published by Innovare Academic Sciences Pvt Ltd. This is an open-access article under the CC BY license (http://creativecommons.org/licenses/by/4.0/) DOI: http://dx.doi.org/10.22159/ijap.2019v11i5.33592

\section{INTRODUCTION}

Psoriasis is a consistently recurring autoimmune disorder of skin characterized by relapsing episodes of inflammatory lesions and hyperkeratotic plaques on the skin with globally occurrence of around $2-5 \%$. The classification of psoriasis was done on the basis of extent of inflammation on skin, localization of rash, severity of the patient condition, and other. Amongst these, the occurrence of chronic plaque psoriasis (CPP) was major in occurrence proportion with equivalent likelihood in both sexes and early onset before the age of 35 y [1]. Psoriasis is a disease caused by a multitude of both genetic and environmental factors such as trauma, drugs, infection, alcohol, smoking and stress but its accurate origin is still not known [2]. Among the currently available treatments including topical therapy, phototherapy and systemic therapy, none of the treatment for psoriasis was found to be safe, effective and able to completely cure the disease [3]. The lack of effective and safe treatment for psoriasis paved a way to develop and implement novel approach with a view to make the therapy more useful and acceptable. Unlike normal skin, pathological progression of psoriasis was supposed to depend on a multitude of coherent events involving the activation of circulating immune cells and their secreted signaling molecules like cytokines, chemokines and growth factors. All these events further progress to mark hyperkeratosis, congealing of epidermis and neovascularization [5]. Currently, Glucocorticoids (GCs) are highly effective for the treatment of inflammatory diseases. It is currently approved for topical use in different dosage forms such as cream, solution, and ointment [6]. The Reported Adverse Drug Reactions (ADRs) with conventional dosage forms include atrophy of skin, steroid acne, hypopigmentation around the application site, allergic contact dermatitis, Cushing like syndrome and osteonecrosis [7]. SLNs have been widely used for topical delivery due to their effective interaction with skin layers, and enhance skin permeation. SLNs were formulated by using different techniques such as highspeed homogenization and ultrasonication etc., [8]. SLNs were comprised of biocompatible solid lipid, surfactant and drug. The drug is usually loaded in the matrix of lipid which is coated by a nontoxic surfactant and in the form of solid dose at room and body temperatures [9]. The suitability of bio-degradable lipids for dermatological applications was also confirmed by successful formulation of lipid-based delivery systems for the management of dermal disorders [10].

The aim of the present work was to develop and optimize solid lipid nanoparticles using design expert software and to explore the in vitro characterization of the optimized formulation for particle size, poly dispersibility index (PDI), percentage entrapment efficiency (\% EE), transmission electron microscopy, differential scanning calorimetry (DSC) and drug release studies. The impact of the carrier type on various critical attributes of a drug such as extent of CP delivery into the epidermis and dermis via an in vitro method for utilizing UV spectroscopy and stability of the developed formulation was also evaluated.

\section{MATERIALS AND METHODS}

Clobetasol-17-propionate was purchased from Yarrow Chemicals (Mumbai, India). Compritol 888 ATO was a kind gift from Gattefossé (France). Tween 80 as a surfactant, poloxamer 188 as a stabilizer, Methanol, and Acetone, were purchased from Sigma-Aldrich (India). Distilled water was obtained from the in-house distillation system.

\section{Preparation of CP-loaded SLNs}

SLNs were prepared by the emulsification-homogenisation method. Formulation procedure was divided into two parts in which one part contained drug and lipid, while the second part contained an aqueous solution of surfactant and stabilizer. Drug and lipid mixture was melted $5{ }^{\circ} \mathrm{C}$ above the melting point of lipid. Aqueous phase was heated to same temperature as like lipid phase. When both parts attain equilibrium, the aqueous phase was incorporated into lipid 
phase and emulsified using high-speed homogenizer (IKA ${ }^{\circledR}$ T-10 basic Ultra-Turrax ${ }^{\circledR}$, Germany) at $10,000 \mathrm{rpm}$ for $15 \mathrm{~min}$. The obtained emulsion was ultrasonicated using a probe Sonicator (VibracellTM 700W; Sonics, USA) at $80 \%$ amplitude and duration of 5 min. Cycles were repeated alternately until it attains uniform particle size. The resulting nanoemulsion was cooled down in an ice bath to produce nanoparticle dispersion. Different formulations were prepared by varying the critical process variables [11].

\section{Experimental design}

In the present work, $3^{3}$ factorial designs were chosen for the optimization of CP SLN using Design-Expert software (Trial Version 11, Stat Ease Inc., and USA). 3 factors were chosen and the responses were measured at 3 levels. Totally 30runs were designed randomly. The design consisted of replicated center points and a set of points lying at the midpoints of each edge of the multidimensional cube. This defines the region of interest used to evaluate the main effects, interaction effects, and quadratic effects of the formulation ingredients, and to optimize the formulation. The quadratic model generated by the design was:

$\mathrm{Y}=\mathrm{A}_{0}+\mathrm{A}_{1} \mathrm{X}_{1}+\mathrm{A}_{2} \mathrm{X}_{2}+\mathrm{A}_{3} \mathrm{X}_{3}+\mathrm{A}_{4} \mathrm{X}_{1} \mathrm{X}_{2}+\mathrm{A}_{5} \mathrm{X}_{2} \mathrm{X}_{3}+\mathrm{A}_{6} \mathrm{X}_{1} \mathrm{X}_{2}+\mathrm{A}_{7} \mathrm{X}_{1}{ }^{2}+\mathrm{A}_{8} \mathrm{X}_{2}{ }^{2}+\mathrm{A}_{9} \mathrm{X}_{3}{ }^{2}$

In which $\mathrm{Y}$ is the measured response of the dependent variables associated with each factor-level combination; $A_{0}-A_{9}$ are the regression coefficients of the respective variables and their interaction terms computed from the observed experimental values of the measured response. $X_{1}-X_{3}$ are the codes for independent variables. Independent variables selected were a concentration of drug: lipid $\left(\mathrm{X}_{1}\right)$, concentration of surfactant $\left(\mathrm{X}_{2}\right)$ and the high-speed homogenization $\left(X_{3}\right)$. Independent variables were represented by level-1, 0 and +1 correspondings to the low, middle, and high values respectively. The measured responses $Y_{1}=$ particle size $(\mathrm{PS})$ and $\mathrm{Y}_{2}=$ Polydispersity index and $Y_{3}=$ entrapment efficiency (\%EE) with constraints were described in [table 1].

Table 1: Selection of independent variables and their levels chosen for the execution of $3^{3}$ factorial designs

\begin{tabular}{llll}
\hline Types of variable independent variable & Levels & Medium (0) & High (+1) \\
\cline { 2 - 4 } & Low (-1) & $1: 4$ & $1: 5$ \\
\hline X1=Drug: lipid Ratio(D: L)(\%w/w) & $1: 3$ & 3 & 4 \\
X2=Surfactant concentration(S)(\%w/w) & 2 & 10 & 15 \\
X3=HSH Time (HT) (min) & 5 & & \\
Dependent variable & & \\
Y1=Particle Size(nm), Y2=Poly Dispersibility Index, Y3=\%Entrapment Efficiency & & \\
\hline
\end{tabular}

$3^{3}$ factorial designs were employed for the study. Further 3D response surface plotted for depicting the effects of the predetermined factors on the measured responses. 3D response surface plots are helpful in explaining the relationship between independent variables and dependent variables. When these plots are carefully observed, the qualitative effect of each variable on each response parameter could be visualized.

\section{Characterization of CP SLNs}

Particle size, polydispersity index and zeta potential measurement

Particle size, polydispersity index (PDI) and zeta potential of the SLNs were measured by dynamic light scattering technique using Malvern Zetasizer Nano ZS90 (Malvern Instruments, UK). CP SLNs colloidal dispersions were diluted 10 times with double distilled water. Particle size and PDI measurements were performed by taking $1 \mathrm{ml}$ of the diluted formulation into polystyrene cuvettes and disposable folded capillary cell for zeta potential at $25^{\circ} \mathrm{C}$,to ensure that the light scattering intensity was within the instruments sensitivity range. Each sample was analyzed in triplicate and the results were shown as mean \pm standard deviation.

\section{$\%$ Entrapment efficiency}

The entrapment efficiency (\%EE) of formulated CP SLN was determined by measuring the concentration of unentrapped drug in the lipidic dispersion by using the centrifugation method. The SLNs Samples were subjected to centrifugation at $10000 \mathrm{rpm}$ for $15 \mathrm{~min}$ (Remi Centrifuge Pvt. Ltd., India) and the amount of Clobetasol propionate Supernatant was collected, suitably diluted with methanol and analyzed for free drug content by UV spectroscopy at $239 \mathrm{~nm}$. \%EE was calculated by following equation.

$$
\% \mathrm{EE}=\frac{\text { Total amount of } \mathrm{CP}-\text { Amount of free } \mathrm{CP}}{\text { Total amount of } \mathrm{CP}} \times 100
$$

Each value was measured in triplicate. The results are shown as mean \pm standard deviation.

\section{Differential scanning calorimetry (DSC) analysis}

DSC analysis was done to predict the compatibility between drug and excipients. Blank SLNs, drug-loaded SLNs and physical mixtures were subjected to DSC analysis (V24.9 build 121, TA instrument, USA). Briefly, blank SLNs and CP SLNs samples were lyophilized prior to DSC analysis. Samples (4-5 mg) were kept in the standard aluminum pans and sealed. Then the pans were placed under the isothermal condition at $25 \pm 1{ }^{\circ} \mathrm{C}$ for $10 \mathrm{~min}$. DSC analysis was performed at $10{ }^{\circ} \mathrm{C} / \mathrm{min}$ from 25 to $300{ }^{\circ} \mathrm{C}$ under a nitrogen atmosphere at a flow rate of $50 \mathrm{ml} / \mathrm{min}$. An empty aluminum sealed pan was used as a reference.

\section{Transmission electron microscopy}

The morphological characters of CP loaded SLNs were observed with transmission electron microscopy (TEM; Philips, Tecnai 20, Holland). A drop of the diluted sample was placed on the surface of carboncoated copper grid and stained with a drop of $1 \%(\mathrm{w} / \mathrm{w})$ aqueous solution of phosphotungstic acid (negative stain) for 30s. Excessive staining solution was washed out by filter paper and a thin aqueous film was left on the surface. After staining, samples were dried at room temperature for $10 \mathrm{~min}$ to perform out investigation [16].

\section{In vitro release study}

In vitro release studies of CP loaded SLNs and plain CP suspension was carried out using modified Franz diffusion cell with a receptor volume capacity of $12.5 \mathrm{ml}$ using cellulose acetate membrane (MWCO-12 000$14000 \mathrm{Da}$, pore size-2.4 nm, HIMEDIA, Mumbai, India) and PBS of $\mathrm{pH}$ 7.4 as a dialyzing medium. The membrane was soaked in doubledistilled water for about $12 \mathrm{~h}$, prior to mounting the membrane (diffusion area $1.95 \mathrm{~cm}^{2}$ ) in the Franz diffusion cell. The pretreated membrane was placed on the modified Franz diffusion cell filled with phosphate buffer (pH7.4) in the receptor compartment. The whole assembly was placed on the magnetic stirrer at $350 \mathrm{rpm}$ and temperature was maintained at $32.0 \pm 0.5^{\circ} \mathrm{C}$. The samples (plain drug suspension and CP loaded SLNs dispersion) equivalent to $2 \mathrm{mg}$ drug were kept over the membrane in donor compartment and stirred. Samples were withdrawn from the receptor compartment at predetermined time intervals $(0,6,8,10,12$ and $24 \mathrm{~h})$ and the same volume was refilled with diffusion medium. The samples were analyzed using UV spectrophotometer at $239 \mathrm{~nm}$ after appropriate dilutions. Percent drug release was calculated and graph was plotted between percent drug releases against time. Release studies were performed in triplicate for each formulation.

\section{Ex-vivo diffusion study}

Ex-Vivo diffusion studies were carried out to evaluate the distribution of CP delivered through CP loaded SLNs suspension and plain CP suspension using sheepskin. Sample of sheepskin was 
obtained from the local slaughterhouse (Tirupati, Andhra Pradesh). The skin diffusion studies were performed using the Franz diffusion cells according to this method. Skin samples were separately treated with the formulations (plain CP suspension and CP loaded SLNs) containing drug equivalent to $400 \mathrm{mg}(6 \mathrm{~h}$ with each test formulation). At the end of the experiment, the skin samples were washed and removed from the Franz cells. Epidermal and dermal layers were manually separated using tweezers. CP was extracted in $10 \mathrm{ml}$ methanol by homogenization of each skin layer and extracted samples were assayed for CP concentration using UV spectroscopy at $239 \mathrm{~nm}$ [12].

\section{Stability studies}

The stability of the developed CP SLNs formulation was evaluated for 3 mo with reference to ICH guidelines and samples were stored in sealed amber colored glass vials at $4{ }^{\circ} \mathrm{C}$. After $3 \mathrm{mo}$, the samples were characterized with respect to particle size, ZP, PDI, EE, and drug loading.

\section{RESULTS AND DISCUSSION}

\section{Statistical analysis of formulations}

Results obtained from all the formulations were analyzed using Design-Expert ${ }^{\circledR}$ Version 11.0 software [table 2]. Response surface plots were generated using the software and new formulations with desired responses were obtained by a numerical optimization technique. The responses obtained were used to study the effect of independent variables. A quadratic model was suggested for particle size, PDI, \% entrapment efficiency. The list of solutions suggested by the software was sorted in the descending order of the desirability and the solution which fulfills the required criteria was reported. The factors which had a significant effect on the responses were identified using Analysis of variance (ANOVA). To evaluate the chosen experimental design, the optimized formulation was prepared as per the solutions obtained and subjected to evaluation parameters. The resulting experimental values were compared with predicted values.

Table 2: Layout of $3^{3}$ full factorial design showing the values of dependent variables of 30 possible CP SLNS formulations

\begin{tabular}{|c|c|c|c|c|c|c|}
\hline Run & X1 & $\mathrm{X} 2$ & X3 & Y1 & Y2 & Y3 \\
\hline 1 & 5 & 3 & 5 & $245.2 \pm 3.21$ & $0.328 \pm 0.061$ & $70.9 \pm 3.11$ \\
\hline 2 & 4 & 3 & 15 & $173.4 \pm 2.23$ & $0.352 \pm 0.025$ & $52.6 \pm 2.63$ \\
\hline 3 & 3 & 4 & 15 & $251.7 \pm 4.21$ & $0.432 \pm 0.039$ & $39.8 \pm 3.12$ \\
\hline 4 & 4 & 2 & 10 & $168.3 \pm 1.11$ & $0.222 \pm 0.042$ & $68.3 \pm 2.58$ \\
\hline 5 & 3 & 3 & 5 & $221.9 \pm 3.55$ & $0.307 \pm 0.064$ & $55.7 \pm 2.64$ \\
\hline 6 & 4 & 3 & 10 & $140.2 \pm 3.71$ & $0.212 \pm 0.023$ & $75.3 \pm 3.13$ \\
\hline 7 & 5 & 3 & 10 & $200.9 \pm 5.63$ & $0.284 \pm 0.035$ & $78.1 \pm 3.45$ \\
\hline 8 & 3 & 4 & 10 & $212.2 \pm 3.08$ & $0.309 \pm 0.045$ & $52.7 \pm 3.63$ \\
\hline 9 & 4 & 3 & 10 & $135.3 \pm 2.05$ & $0.189 \pm 0.083$ & $77.1 \pm 3.86$ \\
\hline 10 & 4 & 4 & 10 & $225.1 \pm 1.32$ & $0.321 \pm 0.057$ & $55.8 \pm 2.45$ \\
\hline 11 & 5 & 2 & 15 & $246.7 \pm 2.56$ & $0.445 \pm 0.062$ & $58.8 \pm 3.12$ \\
\hline 12 & 4 & 2 & 15 & $183.9 \pm 2.05$ & $0.404 \pm 0.039$ & $48.2 \pm 2.69$ \\
\hline 13 & 5 & 2 & 10 & $230.6 \pm 4.12$ & $0.262 \pm 0.056$ & $72.2 \pm 3.56$ \\
\hline 14 & 3 & 4 & 5 & $280.2 \pm 3.98$ & $0.373 \pm 0.066$ & $47.2 \pm 4.12$ \\
\hline 15 & 3 & 2 & 10 & $189.2 \pm 4.21$ & $0.247 \pm 0.089$ & $59.71 \pm 3.23$ \\
\hline 16 & 4 & 2 & 5 & $241.1 \pm 3.26$ & $0.295 \pm 0.056$ & $57.5 \pm 2.36$ \\
\hline 17 & 5 & 4 & 5 & $306.8 \pm 4.25$ & $0.395 \pm 0.063$ & $60.7 \pm 3.65$ \\
\hline 18 & 3 & 3 & 10 & $175.4 \pm 2.35$ & $0.251 \pm 0.052$ & $64.1 \pm 4.10$ \\
\hline 19 & 4 & 4 & 15 & $262.2 \pm 3.97$ & $0.457 \pm 0.061$ & $46.3 \pm 3.25$ \\
\hline 20 & 4 & 3 & 10 & $140.5 \pm 2.18$ & $0.235 \pm 0.073$ & $74.5 \pm 2.53$ \\
\hline 21 & 4 & 3 & 5 & $202.6 \pm 4.91$ & $0.257 \pm 0.069$ & $65.1 \pm 3.22$ \\
\hline 22 & 4 & 4 & 5 & $286.3 \pm 4.72$ & $0.38 \pm 0.056$ & $51.2 \pm 4.12$ \\
\hline 23 & 4 & 3 & 10 & $133.3 \pm 3.66$ & $0.179 \pm 0.081$ & $78.1 \pm 1.11$ \\
\hline 24 & 3 & 3 & 15 & $190.2 \pm 2.15$ & $0.377 \pm 0.071$ & $44.3 \pm 4.23$ \\
\hline 25 & 5 & 2 & 5 & $288.7 \pm 4.13$ & $0.326 \pm 0.082$ & $63.2 \pm 3.22$ \\
\hline 26 & 5 & 4 & 10 & $240.6 \pm 3.23$ & $0.300 \pm 0.071$ & $66.6 \pm 2.12$ \\
\hline 27 & 5 & 4 & 15 & $252.0 \pm 2.06$ & $0.440 \pm 0.065$ & $54.5 \pm 2.81$ \\
\hline 28 & 5 & 3 & 15 & $224.0 \pm 3.22$ & $0.401 \pm 0.059$ & $60.1 \pm 3.24$ \\
\hline 29 & 3 & 2 & 15 & $190.1 \pm 4.89$ & $0.425 \pm 0.063$ & $42.2 \pm 3.88$ \\
\hline 30 & 3 & 2 & 5 & $261.5 \pm 5.01$ & $0.310 \pm 0.077$ & $53.1 \pm 3.26$ \\
\hline
\end{tabular}

*All values are expressed as mean $\pm \mathrm{SD}, \mathrm{n}=3$

\section{Effect on particle size}

The particle size varied for all the formulations were ranging between $133.3 \mathrm{~nm}$ (formulation 23) to $306.8 \mathrm{~nm}$ (formulation $17)$ at various factor-level combinations. The independent factors (variables) affecting the particle size were concentration of lipid, concentration of surfactant and hominization speed on particle size was studied, and the responses obtained are given in [table 2]. The suggested quadratic model with F-value of 39.03 implies that the model is significant. The model P-value was $<0.0001$ indicated that the model terms are significant. $\mathrm{P}$ values for, lipid to drug ratio was 0.0002 , surfactant concentration was $<0.0001$ and that for hominization time was $<0.0001$. These results indicated there was no significant affect in particle size with the change in independent variables. The regression coefficient value $\mathrm{R}^{2}$ was 0.9461 , adjusted $\mathrm{R}^{2}$ was 0.9219 and predicted $R^{2}$ was 0.8652 indicating minimum variations in the experimental model. The adequate precision ratio, measuring the signal to noise ratio was 20.407 indicating an adequate signal. A ratio greater than 4 is desirable, and this model can be used to navigate the design space. The polynomial equation in terms of coded factors is given below.

Particle size: $145.83+14.62 \mathrm{~A}+17.61 \mathrm{~B}-20.01 \mathrm{C}-5.82 \mathrm{AB}+1.13 \mathrm{AC}+$ $5.27 \mathrm{BC}+27.69 \mathrm{~A}^{2}+45.91 \mathrm{~B}^{2}+44.46 \mathrm{C}^{2}$

The individual factor A, lipid to drug ratio had a positive effect on particle size as showed by the positive coefficient estimate value. The particle size increased with the ratio of lipid to the drug, whereas surfactant concentration showed a positive effect and homogenization time showed a negative effect. The combined effect of lipid to drug ratio and surfactant concentration was negative, whereas the homogenization time with lipid to drug ratio and surfactant concentration showed a positive effect. An increase in the particle size was observed with a concomitant increase in the proportion of lipid and reduction in the surfactant concentration. Larger particle size with 5:1 lipid to drug ratio could be attributed to the increased viscosity of the system due to higher lipid 
concentration. Another possible explanation to this may be a film of loosely arranged surfactant molecules at the interface of the two layers in Nano dispersion due to the lower concentration of surfactant in comparison to the lipid. On the other hand, lower lipid with higher surfactant resulted in fine Nano dispersion with smaller sized particles. The size of SLN decreased with increase in homogenization time. This could be due to the shear energy induced by homogenization. The effect of lipid: drug ratio, sonication time and surfactant concentration on the particle size is presented in the form of response surface plots in [fig. 1].
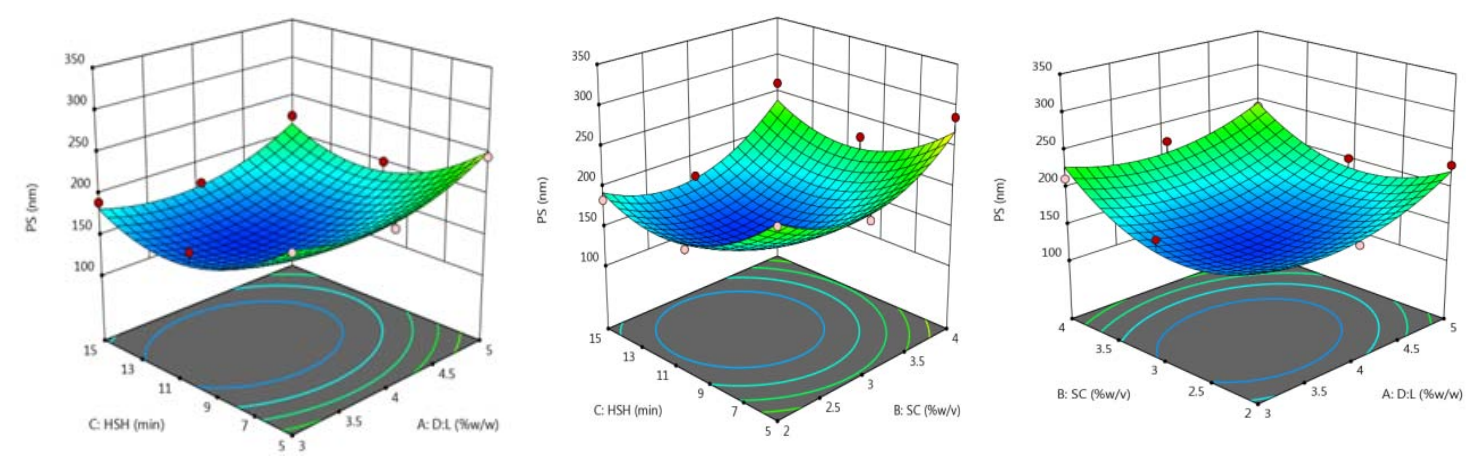

Fig. 1: 3D response surface plot for effect of lipid: drug ratio, homogenization time and surfactant concentration on particle size

\section{Effect on PDI}

The suggested quadratic model with F-value of 44.89 implies that the model is significant. The model P-value was $<0.0001$ indicated that the model terms are significant. $P$ values for, lipid to drug ratio was 0.1083 , surfactant concentration was $<0.0001$ and that for hominization time was $<0.0001$ the results indicated there was no significant difference in PDI with the change in hominization time. The regression coefficient value $\mathrm{R}^{2}$ was 0.9528 , adjusted $\mathrm{R}^{2}$ was 0.9316 and predicted $R^{2}$ was 0.9059 indicating minimum variations in the experimental model. The adequate precision ratio, measuring the signal to noise ratio was 20.160 indicating an adequate signal. A ratio greater than 4 is desirable, and this model can be used to navigate the design space. The polynomial equation in terms of coded factors is given below.
PDI: $\quad 0.2131+0.008 \mathrm{~A}+0.026 \mathrm{~B}+0.042 \mathrm{C}-0.002 \mathrm{AB}-0.0006 \mathrm{AC}-0.013 \mathrm{BC}+$ $0.026 \mathrm{~A}^{2}+0.048 \mathrm{~B}^{2}+0.1089 \mathrm{C}^{2}$

As per the polynomial equation, the effect of lipid to drug ratio, surfactant concentration and homogenization time was shown a positive effect. All the interaction effects showed a negative coefficient estimate value indicating the indirect relationship with the PDI. It was observed that when $4 \% \mathrm{w} / \mathrm{w}$ tween 80 was used, PDI was increased [Table 2]. This was because; during homogenization process alkyl chain of the surfactant molecule covers the surface of lipid particle via hydrophobic interaction to form a stable lipid matrix. Once this stable matrix is formed, excess surfactant may lead to accumulation of surfactant particles on the surface of stable lipid matrix causing increase in PDI as was observed in the form of response surface plots in [fig. 2].
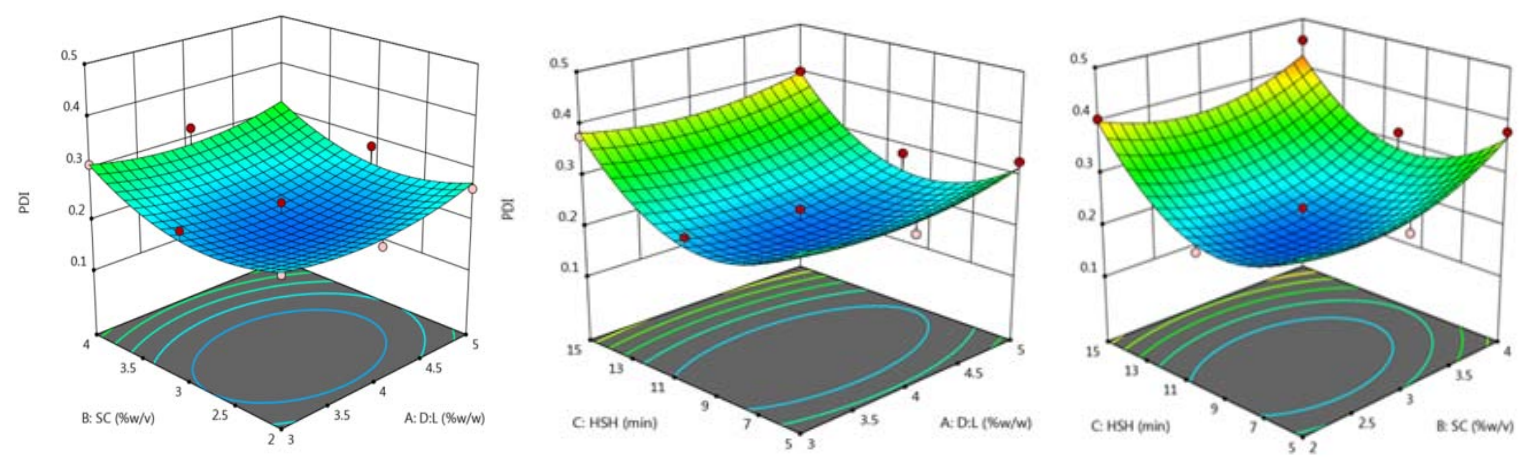

Fig. 2: 3D response surface plot for effect of lipid: drug ratio, homogenisation time and surfactant concentration on polydispersity index

\section{Effect on \% EE}

The suggested quadratic model with F-value of 45.47 implies that the model is significant. The model $\mathrm{P}$ value was $<0.0001$ indicated that the model terms are significant. P values for, lipid to drug ratio was 0.0001 , surfactant concentration was 0.0008 and that for hominization time was $<0.0001$ the results indicated there was no significant difference in \%EE with the change in hominization time. The regression coefficient value $\mathrm{R}^{2}$ was 0.9534 , adjusted $\mathrm{R}^{2}$ was 0.9324 and predicted $\mathrm{R}^{2}$ was 0.8971 indicating minimum variations in the experimental model. The adequate precision ratio, measuring the signal to noise ratio was 25.75 indicating an adequate signal. A ratio greater than 4 is desirable, and this model can be used to navigate the design space. The polynomial equation in terms of coded factors is given below.
Entrapment efficiency (\%): $73.28+7.02 \mathrm{~A}-2.69 \mathrm{~B}-4.32 \mathrm{C}+0.242 \mathrm{AB}+$ $0.691 \mathrm{AC}+0.508 \mathrm{BC}-0.911 \mathrm{~A}^{2}-8.56 \mathrm{~B}^{2}-13.00 \mathrm{C}^{2}$

The results for drug entrapment in solid lipid nanoparticle formulations were shown in table 4 . The term A, lipid to drug ratio showed a positive coefficient estimate value indicating the direct relationship with the entrapment efficiency, whereas surfactant concentration and homogenization time showed a negative effect. The interaction effect of lipid to drug ratio with surfactant concentration and homogenization time showed a positive effect. Formulation with lipid: drug ratio of $4: 1$ showed greater drug entrapment, i.e., $78.1 \%$. A higher \%EE could be due to the presence of higher amount of lipid which provides additional space for a drug molecule to embed in, thereby increasing total surface area. [table 2] This can lead to reduction in the diffusion 
rate of the solute molecule as the viscosity of the lipidic phase is higher and thus showed higher \%EE s compared to others. \% EE was found to increase with the increasing amount of lipid to drug molar ratio. Thus, \% EE was found to be mainly dependent on the drug: lipid ratio of the formulation. [fig. 3] represents the response surface plots depicting the effect of various factors on $\% \mathrm{EE}$.
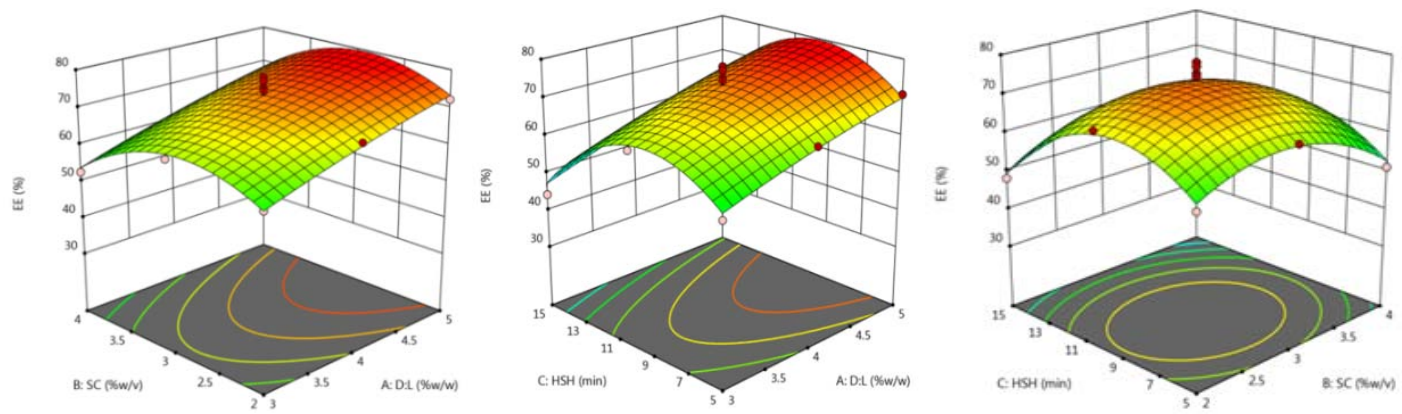

Fig. 3: 3D response surface plot for effect of lipid: drug ratio, homogenisation time and surfactant concentration on entrapment efficiency

\section{Optimization and validation}

The desirability function was investigated using Design-Expert software to acquire the optimized formulation. The set criteria for the optimization were a minimum size of solid lipid nanoparticles, optimum Polydispersibility index, and maximum entrapment efficiency of drug. The solutions generated by the software were sorted in the descending order of the desirability and the formulation with highest desirability factor was considered for further formulation [fig. 4]. The predicted values were compared with actual values by calculating the relative error. The relative error was found to be less than $5 \%$ indicating a minimum variation in the batch [table 3].
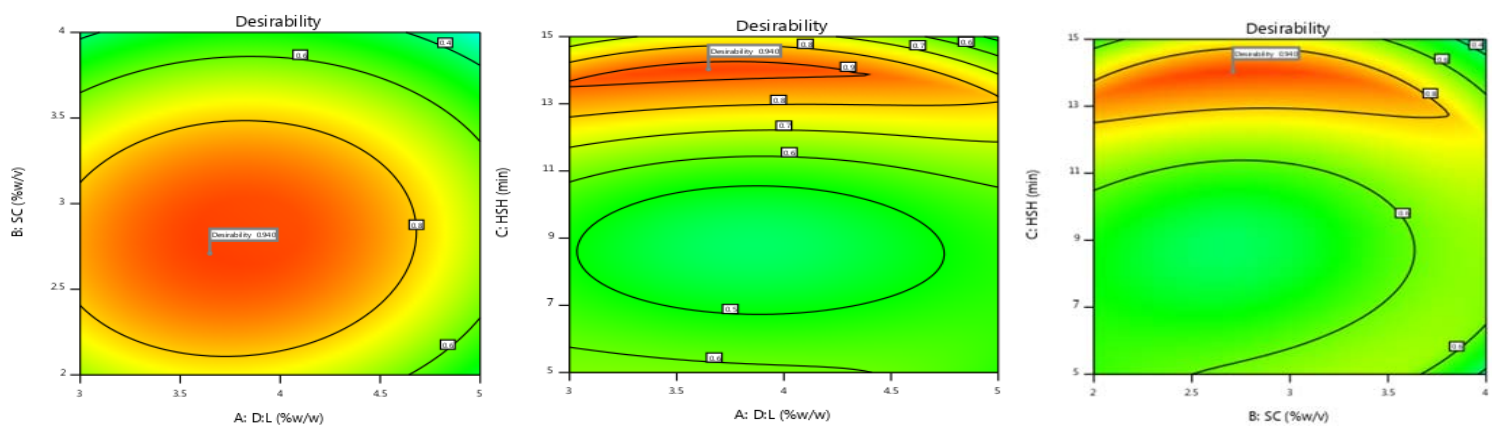

Fig. 4: Desirability for the effect of lipid: drug ratio, homogenisation time and surfactant concentration

Table 3: Predicted and observed responses for the optimized formulation

\begin{tabular}{lllllll}
\hline $\begin{array}{l}\text { Factor 1 } \\
\text { lipid: drug ratio }\end{array}$ & $\begin{array}{l}\text { Factor 2 } \\
\text { surfactant concentration }\end{array}$ & $\begin{array}{l}\text { Factor 3 } \\
\text { HSH }\end{array}$ & Responses & Observed & Predicted & Relative error (\%) \\
\hline 3.64 & 2.71 & 14.03 & Ps(nm) & $153.81 \pm 10.1$ & $155.02 \pm 9.21$ & 1.21 \\
& & & PDI & $0.318 \pm 1.35$ & $0.320 \pm 0.12$ & 0.002 \\
& & \%EE & $58.53 \pm 3.53$ & $60.21 \pm 2.31$ & 1.68 \\
\hline
\end{tabular}

*All values are expressed as mean \pm SD, $\mathrm{n}=3$

\section{Mean particle size, polydispersity index, zeta potential, percent entrapment efficiency}

The mean particle size of optimized CP loaded SLNs was found to be $133.3 \pm 3.66 \mathrm{~nm}$ with PDI to $0.179 \pm 0.081$ [table 2]. The ZP of the optimized formulation was found to be- $36.2 \pm 0.11 \mathrm{mV}$. The zeta potential value of selected batch indicated the stable formulation. High ZP (negative or positive) indicates stable nanoparticle dispersion as highly charged particles are responsible for the prevention of aggregation of the particles due to electric repulsion. $\% \mathrm{EE}$ of the optimized formulation was found to be $78.1 \pm 1.11 \%$. High entrapment can be attributed due to the lipophilic nature of drug having higher affinity for the selected lipid matrix.

\section{Characterization of optimized solid lipid nanoparticle}

\section{Transmission electron microscopy}

Size and shape of the optimized batch of nanoparticles were evaluated by TEM. TEM images of the SLNs confirmed the oval and nearly spherical shape of nanoparticles with narrow size distribution. They further confirmed non aggregation of nanoparticles [fig. 5]. The diameters of the nanoparticles observed in the micrographs were in good agreement with data obtained from Malvern particle size analyzer.

\section{Differential scanning calorimetry}

DSC is a tool to investigate the melting and recrystallization behavior of crystalline material like SLNs 13. Fig. 6 shows the DSC thermograms of pure CP, Compritol 888 ATO, physical mixture of CP and Compritol 888 ATO, and CP loaded SLNs. Compritol 888 ATO exhibited a sharp endothermic event ascribing to the melting point around $75.41^{\circ} \mathrm{C}$ [fig. 6a]. Pure CP showed a sharp endothermic peak at $196.24{ }^{\circ} \mathrm{C}$ corresponding to its melting point, indicating its characteristic crystalline nature [fig. $6 \mathrm{~b}]$. These sharp melting endothermic peaks of bulk lipid and drug indicated that the starting materials were crystalline. Characteristic peak for CP was completely absent in lyophilized CP loaded SLNs [fig. 6d], while it was clearly evident in 
physical mixture of $\mathrm{CP}\left(196.24^{\circ} \mathrm{C}\right)$ and Compritol 888 ATO $75.41^{\circ} \mathrm{C}$ as showed in [fig. 6c]. It has been reported that when the drug does not show its endothermic peak in the CP SLNs formulations, it is said to be in the amorphous state [14]. Hence, it could be concluded that the drug was present in the amorphous phase and might have been homogeneously dispersed in the SLNs.
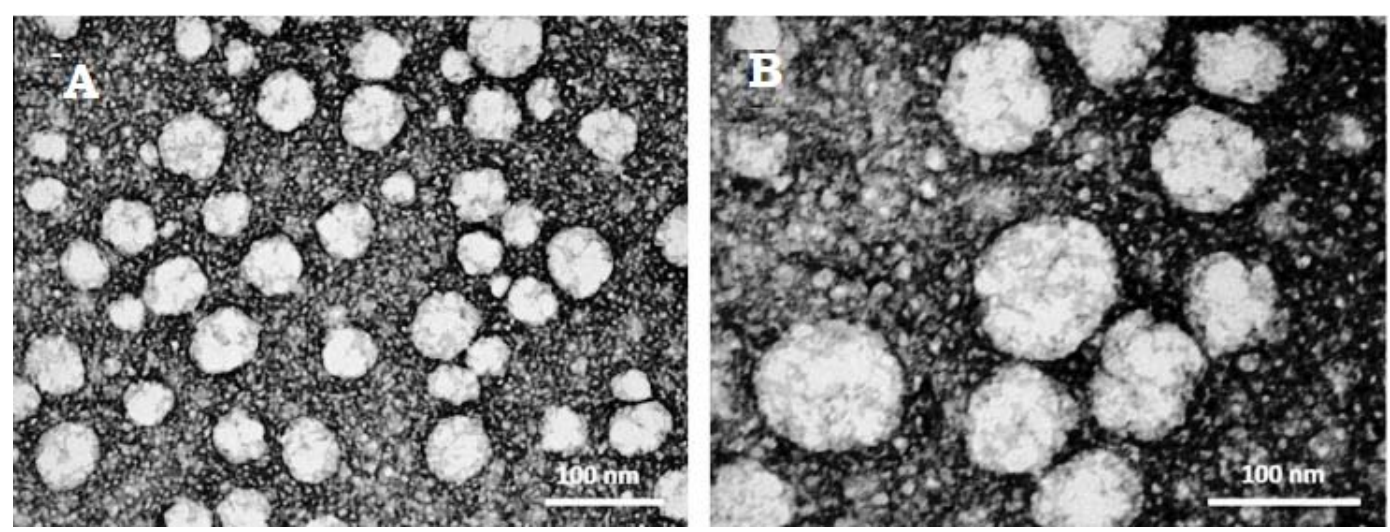

Fig. 5: CP loaded SLNs (formulation no. 23) visualized by transmission electron microscopy. (a) Overall view showing particle polydispersity, (b) magnified view showing particle internal structure

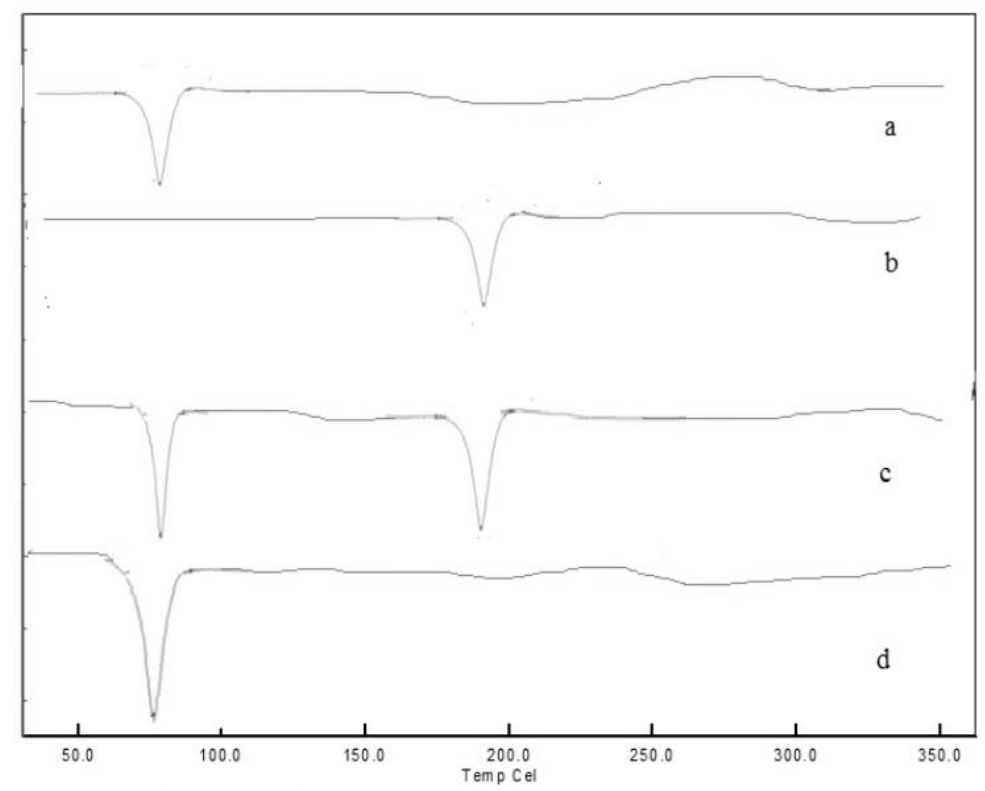

Fig. 6: DSC study. (a) Pure Compritol 888, (b) pure CP, (c) physical mixture of CP and Compritol 888 ATO (d) CP loaded SLNs

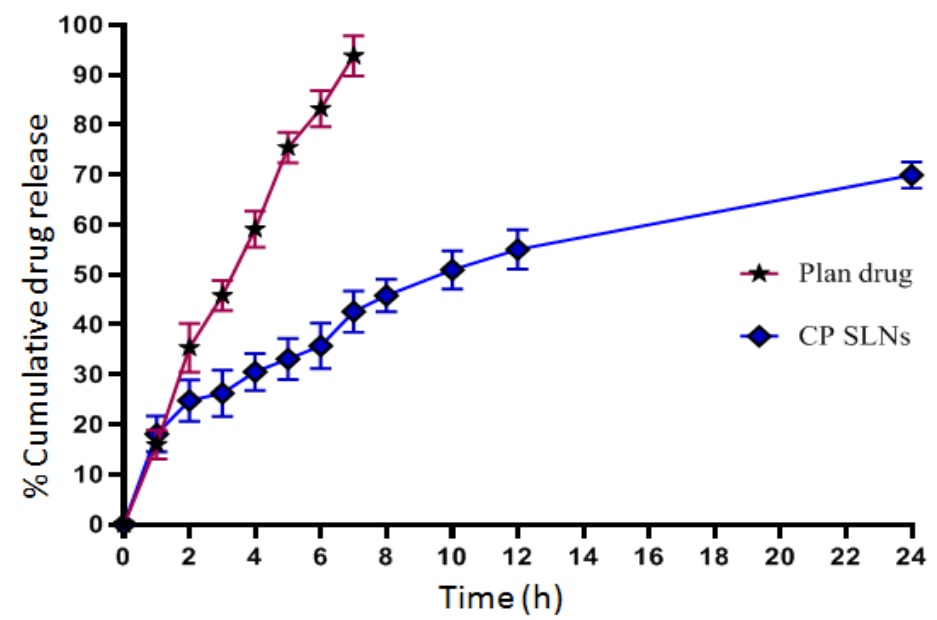

Fig. 7: In vitro drug release profile from pure CP suspension and CP loaded SLNs suspension in phosphate buffer (pH 7.4), ${ }^{*}$ All values are expressed as mean $\pm S D, n=3$ 


\section{In vitro release study}

The cumulative percentage release of $\mathrm{CP}$ from plain $\mathrm{CP}$ suspension and CP loaded SLNs suspension was investigated in vitro over a period of $24 \mathrm{~h}$. Each sample was analyzed in triplicate and release curves have been shown in [fig. 7]. The drug release from plain $\mathrm{CP}$ suspension was faster with $93.77 \pm 4.04 \%$ release of $\mathrm{CP}$ within $7 \mathrm{~h}$. On the other hand CP loaded SLNs suspension depicted a biphasic release pattern with relatively faster initial burst release followed by comparatively slower release with $69.90 \pm 2.60 \%$ release of CP after $24 \mathrm{~h}$. Burst release can be useful to improve the penetration of the drug, while sustained-release supplies the drug over a prolonged period of time. Regarding the drug release profiles, CP loaded SLNs and plain CP suspension followed Higuchi release kinetics $\left(\mathrm{r}^{2}=\right.$ $0.989)$ and zero-order release kinetics $\left(r^{2}=0.981\right)$, respectively.
Similar results for novel and conventional formulation were obtained [17].

\section{Ex-vivo diffusion study}

The ability of CP loaded SLNs suspension and pure CP suspension to deliver CP across sheepskin was evaluated. UV spectroscopy was used to quantify $\mathrm{CP}$ in the epidermis, dermis, and receptor. As shown in [fig. 8], plain CP suspension delivered the maximum amount of $\mathrm{CP}$ in the receptor compartment $(145.78 \mu \mathrm{g} / \mathrm{ml})$ with minimum amount of CP in the epidermis $(19.12 \mu \mathrm{g} / \mathrm{ml})$ and dermis $(36.56 \mu \mathrm{g} / \mathrm{ml})$ when examined though $1 \mathrm{~cm}^{2}$ diffusion areas. This indicated maximal distribution of drug in systemic circulation after $6 \mathrm{~h}$. At the same time CP loaded SLNs suspension delivered its maximum amounts of CP into the epidermis $(48.22 \mu \mathrm{g} / \mathrm{ml})$, which is the location of the over-proliferation of keratinocytes and development of psoriasis.

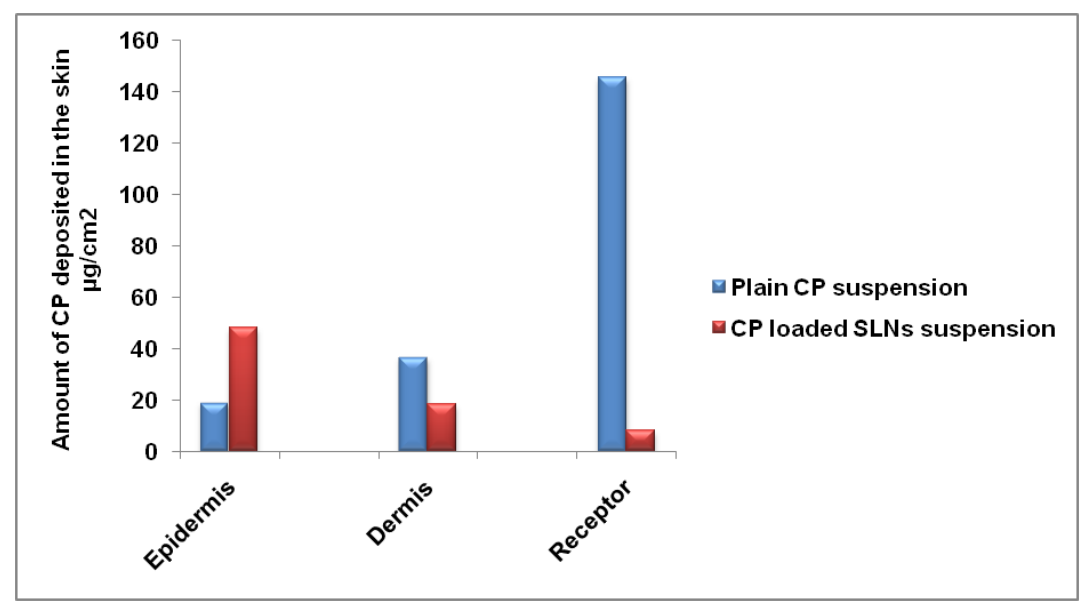

Fig. 8: In vitro skin distribution study of pure CP loaded suspension and CP loaded SLNs

Moreover, the minimum amount of $\mathrm{CP}$ was found to be present in the dermis $(18.34 \mu \mathrm{g} / \mathrm{ml})$ and in the receptor compartment $(8.16 \mu \mathrm{g} / \mathrm{ml})$ of the Franz cell, demonstrating the potential of CP loaded SLNs to limit systemic escape of the steroidal drug. In addition, the results of skin distribution study demonstrated that CP loaded SLNs suspension possesses the ability to efficiently deliver the drug with limited systemic escape and could be considered as an appropriate carrier for topical therapy of psoriasis. Based on skin distribution data reported that CP delivery to the skin could be related to the increased solubility of drug in the lipid matrix (in case of SLNs) which in turn results into high concentration gradient at the skin surface [15]. It is assumed that due to the lipid-rich environment of the epidermis, lipophilic compounds, like $\mathrm{CP}$, may remain a drug depot in the intercellular space of epidermal cells, allowing slow drug permeation into the lower viable epidermis where psoriasis originates. In the SLN based formulation, CP largely resides in the epidermis (topical delivery) because of the enhanced hydrophilicity of the epidermis which limits significant partitioning of hydrophobic drug. Moreover, it is wellknown that skin in diseased condition, particularly in case of psoriatic skin, becomes more permeable than healthy skin. Hence, inpatients, more deposition of CP in the epidermis is expected which makes CP loaded SLNs more effective.

\section{Stability study}

Stability studies were carried out according to assess the stability and integrity of CP loaded SLNs [11]. The CP loaded SLNs showed minor enhancement of particle size and PDI with a slight reduction of ZP and \%EE after 3 mo storage at $4{ }^{\circ} \mathrm{C}$ Percent change in PS and \%EE were $2.91 \%$ and $3.15 \%$ respectively on 3 mo storage of SLNs formulation. The changes were insignificant which indicates excellent physical stability of the SLNs during their storage at $4{ }^{\circ} \mathrm{C}$ for $3 \mathrm{mo}$.

\section{CONCLUSION}

CP loaded SLNs were prepared using Compritol 888 ATO as lipid matrix, poloxamer-188 as stabilizer employing the emulsification- homogenisation technique. The important parameters like drug: lipid ratio, surfactant concentration and homogenization time were optimized by $3^{3}$ full factorial design to obtain a minimal PS, Narrow PDI and highest \% EE. Thus, desirable goals could be achieved by systematic formulation approach the shortest possible time with reduced number of experiments, The maximum zeta potential of$36.2 \pm 0.11 \mathrm{mV}$ was attributed to the anionic nature of the lipid matrix and indicated, physical stability of the CP SLNs. DSC studies confirmed the absence of any interaction between $\mathrm{CP}$ and lipids. TEM imaging of CP loaded SLNs exhibited spherical shape of SLNs. Drug release from SLNs dispersion and CP suspension showed a sustained release of the drug over a prolonged period of time as compared to free drug. Drug release from SLNs based suspension followed Higuchi model. Stability studies indicate a significant change in PS, when stored at room temperature.

\section{ACKNOWLEDGMENT}

The authors are thankful to the principal and management of Krishna Teja Pharmacy College, for providing necessary facilities to carry out this work.

\section{AUTHORS CONTRIBUTIONS}

All the authors have contributed equally

\section{CONFLICT OF INTERESTS}

Declared none

\section{REFERENCES}

1. Pradhan M, Singh D, Sing MR. Novel colloidal carriers for psoriasis: current issues, mechanistic insight and novel delivery approaches. J Controlled Release 2013;170:380-95.

2. Suruta T. NF-kappa B links keratinocytes and lymphocytes in the pathogenesis of psoriasis. Recent Pat Inflamm Allergy Drug Discovery 2009;3-1:40-8. 
3. Krueger J, Bowcock A. Psoriasis pathophysiology: current concepts of pathogenesis. Ann Rheum Dis 2005;64(Suppl 2):30-6.

4. Mabuchi T, Chang TW, Quinter S. Chemokine receptors in the pathogenesis and therapy of psoriasis. J Dermatol Sci 2012;65:4-11.

5. Nickoloff BJ, Nestle FO. Recent insights into the immunopathogenesis of psoriasis provide new therapeutic opportunities. J Clin Invest 2004;113:1664-75.

6. Senyigit T, Sonvico F, Barbieri S, Ozer O, Santi P, Colombo P. Lecithin/chitosan nanoparticles of clobetasol-17-propionate capable of accumulation in pig skin. J Controlled Release 2010;142:368-73.

7. Patel HK, Barot BS, Parejiya PB, Shelat PK, Shukla A. Topical delivery of clobetasol propionate loaded microemulsion based gel for effective treatment of vitiligo: ex vivo permeation and skin irritation studies. Colloids Surf B Biointerfaces 2013;102:86-94.

8. Seyyed SR, Jafar A, Majid S, Katayoun MS, Ali N. Topical gel of metformin solid lipid nanoparticles: a hopeful promise as a dermal delivery system. Colloids Surfaces B: Biointerfaces 2019;175:150-7.

9. Rainer H Muller, Karsten Mader, Sven Gohla. Solid lipid nanoparticles (SLN) for controlled drug delivery-a review of the state of the art. Eur J Pharm Biopharm 2000;50:161-77.

10. Pauporte M, Maibach H, Lowe N, Pugliese M, Friedman DJ, Mendelsohn $\mathrm{H}$, et al. Fluocinolone acetonide topical oil for scalp psoriasis. J Dermatol Treat 2004;15:360-4.

11. Das S, Ng WK, Kanaujia P, Kim S, Tan RB. Formulation design, preparation and physicochemical characterizations of solid lipid nanoparticles containing a hydrophobic drug: effects of process variables. Colloids Surf B: Biointerfaces 2011;88:483-9.

12. Khurana S, Bedi PM, Jain NK. Preparation and evaluation of solid lipid nanoparticles based nanogel for dermal delivery of meloxicam. Chem Phys Lipids 2013;175:65-72.

13. Mingxing Liu, Jing Dong, Yajiang Yang, Xiangliang Yang, Huibi $\mathrm{Xu}$. Characterization and release of triptolide-loaded poly $(\mathrm{d}, \mathrm{l}-$ lactic acid) nanoparticles. Eur Polymer J 2005;41:375-82.

14. Agrawal Y, Petkar KC, Sawant KK. Development, evaluation and clinical studies of Acitretin loaded nanostructured lipid carriers for topical treatment of psoriasis. Int J Pharm 2010;401:93-102.
15. Kilfoyle BE, Sheihet L, Zhang Z, Laohoo M, Kohn J, Michniak Kohn BB. Development of paclitaxel-tyro spheres for topical skin treatment. J Controlled Release 2012;163:18-24.

16. Rawat M, Saraf S, Saraf S. Influence of selected formulation variables on the preparation of enzyme-entrapped eudragit S100 microspheres. AAPS PharmSciTech 2007;8:116.

17. Harshad V, Abhinesh K, Krutika Sawant. Development of solid lipid nanoparticles based controlled release system for topical delivery of terbinafine hydrochloride. Eur J Pharm Sci 2013;49:311-22.

18. Guttman Yassky E, Krueger JG. Psoriasis: evolution of pathogenic concepts and new therapies through phases of translational research. Br J Dermatol 2007;157:3-15.

19. Priyanka T, Animesh K, Pavan Kumar Jain, Jay Ram Patel. Carbomer gel bearing methotrexate loaded lipid nanocontainers shows improved topical delivery intended for effective management of psoriasis. Int J Biol Macromol 2018;120:1322-34

20. Swapnil Kumar, Reema Narayan, Vasif Ahammed, Yogendra Nayak, Anup Naha, Usha Y Nayak. Development of ritonavir solid lipid nanoparticles by box behnken design for intestinal lymphatic targeting. J Drug Delivery Sci Technol 2018;44:181-9.

21. Madhulika Pradhan, Deependra Singh, Manju Rawat Singh. Development characterization and skin permeating potential of lipid based novel delivery system for topical treatment of psoriasis. Chem Phys Lipids 2015;186:9-16.

22. Madhu Gupta, Suresh P Vyas. Development, characterization and in vivo assessment of effective lipidic nanoparticles for dermal delivery of fluconazole against cutaneous candidiasis. Chem Phys Lipids 2012;165:454-61.

23. Cornelia M, Keck, Andjelka K, Rainer H, Muller. Formulation of solid lipid nanoparticles (SLN): the value of different alkyl polyglucoside surfactants. Int J Pharm 2014;474:33-41.

24. Kelidari HR, Saeedi M, Akbari J, Morteza Semnani K, Gill P. Formulation optimization and in vitro skin penetration of spironolactone loaded solid lipid nanoparticles. Colloids Surfaces B: Biointerfaces 2015;128:473-9.

25. Sonali B, Yuechao D, Paul Takhistov, Bozena Michniak-Kohn. Formulation optimization and topical delivery of quercetin from solid lipid-based nanosystems. Int J Pharm 2013; 441:56-66. 